\title{
A study of the b-quark fragmentation function with the DELPHI detector at LEP I and an averaged distribution obtained at the $\mathrm{Z}$ pole
}

\author{
Eli Ben-Haim *† \\ LPNHE, IN2P3/CNRS, Université Pierre et Marie Curie-Paris VI \\ E-mail: benhaimein2p3.fr
}

The nature of b-quark jet hadronisation has been investigated using data taken at the $\mathrm{Z}$ peak by the DELPHI detector. Two complementary methods are used to reconstruct the energy of weakly decaying b-hadrons and measure the b-quark fragmentation distribution. They are then averaged to give a single result.

Combining the DELPHI result with those obtained at the Z peak by ALEPH, OPAL and SLD, yields a world average b-quark fragmentation distribution.

The resulting fragmentation distribution is analysed in the framework of two choices for the perturbative contribution (parton shower and Next to Leading Log QCD calculation) in order to extract measurements of the non-perturbative contribution to be used in studies of b-hadron production in other experimental environments than $e^{+} e^{-}$collisions at the $\mathrm{Z}$ peak. In the parton shower framework, data favour the Lund model ansatz and corresponding values of its parameters have been determined within PYTHIA 6.156 from the world average fragmentation distribution:

$$
a=1.48_{-0.10}^{+0.11} \text { and } b=0.509_{-0.023}^{+0.024} \mathrm{GeV}^{-2},
$$

with a correlation factor $\rho=92.6 \%$.

35th International Conference of High Energy Physics - ICHEP2010,

July 22-28, 2010

Paris France

\footnotetext{
*Speaker.

$\dagger$ On behalf of the DELPHI Collaboration
} 


\section{Introduction}

The fragmentation of $a b \bar{b}$ quark pair from $\mathrm{Z}$ decay, into jets of particles including the parent b-quarks bound inside b-hadrons, is a process that can be viewed in two stages. The first stage involves the b-quarks radiating hard gluons at scales of $Q^{2} \gg \Lambda_{\mathrm{QCD}}^{2}$ for which the strong coupling is small $\alpha_{s} \ll 1$. These gluons can themselves split into further gluons or quark pairs in a kind of 'parton shower'. By virtue of the small coupling, this stage can be described by perturbative QCD implemented either as exact QCD matrix elements or leading-log parton shower cascade models in event generators. As the partons separate, the energy scale drops to $\sim \Lambda_{\mathrm{QCD}}^{2}$ and the strong coupling becomes large, corresponding to a regime where perturbation theory no longer applies. Through the self interaction of radiated gluons, the colour field energy density between partons builds up to the point where there is sufficient energy to create new quark pairs from the vacuum. This process continues with the result that colourless clusters of quarks and gluons with low internal momentum become bound up together to form hadrons. This 'hadronisation' process represents the second stage of the b-quark fragmentation which cannot be calculated in perturbation theory and must be modelled in some way. In simulation programs this is made via a 'fragmentation function' which, in the case of b-hadron production, parameterises how energy/momentum is shared between the parent b-quark and its final state b-hadron.

The purpose of this study is to measure the non-perturbative contribution to b-quark fragmentation in a way that is independent of any non-perturbative hadronisation model. Up to the choice of either QCD matrix elements or leading-log parton shower to represent the perturbative phase, results are obtained that are applicable to any b-hadron production environment in addition to the $\mathrm{Z} \rightarrow \mathrm{b} \overline{\mathrm{b}}$ data on which the measurements were made.

\section{The DELPHI measurement and the averaged distribution at the $Z$ pole}

The DELPHI experiment used two different and complementary approaches to reconstruct the energy of weakly decaying b-hadrons, $E_{\mathrm{B}}^{\text {weak }}$ : Regularised Unfolding and Weighted Fitting [1]. These measurements, presented in terms of $x_{\mathrm{B}}^{\text {weak }}=E_{\mathrm{B}}^{\text {weak }} / E_{\text {beam }}$, are then combined to an average energy distribution. The resulting distribution is shown on the left hand side of Fig 1, and the average value $\left\langle x_{\mathrm{B}}^{\text {weak }}\right\rangle$ is measured to be $0.699 \pm 0.011$.

Other measurements have been obtained at the Z peak by ALEPH, OPAL and SLD [2, 3, 4]. They are also shown on the left hand side of Fig. 1. A combination of all these measurements with the one obtained by DELPHI yields $\left\langle x_{\mathrm{B}}^{\text {weak }}\right\rangle=0.7092 \pm 0.0025$. The full combined "world average" distribution is given in Ref. [1], where the averaging procedure is explained in detail.

\section{Extraction of the non-perturbative QCD component and fits to hadronisation models}

The $x_{\mathrm{B}}^{\text {weak }}$ distribution is then interpreted as the combined result of a perturbative and a nonperturbative part. Corresponding fragmentation functions are determined by:

1. describing the perturbative part with a NLL QCD calculation and using the inverse Mellin transformation to extract the non-perturbative part ; 
2. finding the best fit to the data of the parameters of a given hadronisation model, in the framework of a full simulation of the hadronisation process, where the perturbative contribution is made by a parton shower model.

In both cases, the idea is to extract measurements of the non-perturbative contribution to be used in studies of b-hadron production in other experimental environments than $e^{+} e^{-}$collisions at the $\mathrm{Z}$ pole. In the first method, the extracted non-perturbative component does not depend on any hadronisation model. On the other hand, the non-perturbative distribution depends on the choice of the perturbative component, and has to be used jointly with the adequate one (see Refs. $[1,5]$ for the full study and results). In the parton shower framework, only the Lund and Lund-Bowler models give reasonable description of the data, the Lund ansatz being favoured. The corresponding values of its parameters within PYTHIA $6.156^{1}$, obtained from the world average $x_{\mathrm{B}}^{\text {weak }}$ distribution are:

$$
a=1.48_{-0.10}^{+0.11} \text { and } b=0.509_{-0.023}^{+0.024} \mathrm{GeV}^{-2},
$$

with a correlation factor $\rho=92.6 \%$. This result is shown on the right hand side of Fig. 1
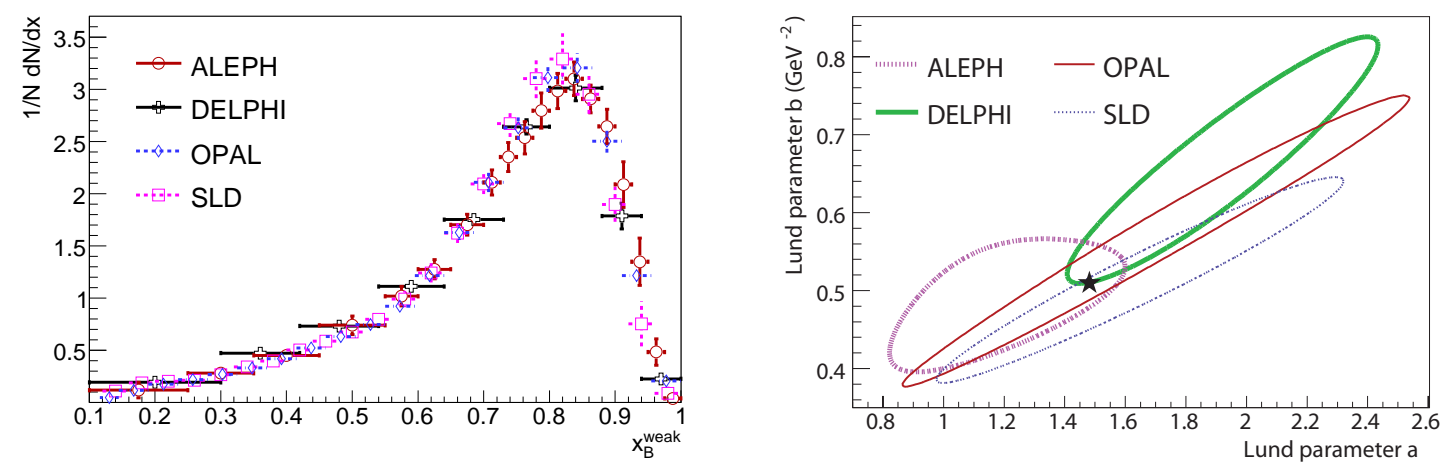

Figure 1: Left: Comparison between the various measurements of the b-quark fragmentation distribution versus $x_{\mathrm{B}}^{\text {weak }}$. Right: Contours of $68.3 \%$ coverage probability for the $a$ and $b$ Lund parameters corresponding to a separate fit to each experiment and the result obtained in the combined fit marked by $\star$.

\section{References}

[1] J. Abdallah et al., DELPHI Collaboration, accepted for publication in Eur. Phys. J. C (2011).

[2] A. Heister et al., ALEPH Collaboration, Phys. Lett. B512 (2001) 30.

[3] G. Abbiendi et al., OPAL Collaboration, Eur.Phys.J. C29 (2003) 463.

[4] K. Abe et al., SLD Collaboration, Phys. Rev. D65 (2002) 092006, Erratum-ibid. D66 (2002) 079905.

[5] E. Ben-Haim et al., Phys. Lett. B580 (2004) 108.

\footnotetext{
${ }^{1}$ Parameters obtained for the non-perturbative component depend on the choice for the perturbative evaluation (e.g. PYTHIA 6.156, JETSET 7.3).
} 